\title{
NONLINEAR EVOLUTION EQUATIONS AND PRODUCT INTEGRATION IN BANACH SPACES
}

BY

G. F. WEBB

\begin{abstract}
The method of product integration is used to obtain solutions to the nonlinear evolution equation $g^{\prime}=A g$ where $A$ is a function from a Banach space $S$ to itself and $g$ is a continuously differentiable function from $[0, \infty)$ to $S$. The conditions required on $A$ are that $A$ is dissipative on $S$, the range of $(e-\varepsilon A)=S$ for all $\varepsilon \geqq 0$, and $A$ is continuous on $S$.
\end{abstract}

1. Introduction. Let $S$ be a Banach space and let $A$ be a mapping from a subset of $S$ to $S$. An evolution equation is a system $g^{\prime}=A(g), g(0)=p$, where $g$ is a continuous function from [0, ) to $S$ and $p$ is a point in $S$. In [3] F. Browder has considered nonlinear evolution equations in which $S$ is the Hilbert space and $A$ is continuous, bounded, and dissipative on $S$. In recent articles Y. Kōmura [12], T. Kato [10], and M. Crandall and A. Pazy [5] have considered nonlinear evolution equations in which $S$ is the Hilbert space and $A$ is maximal dissipative, not necessarily continuous, and is the infinitesimal generator of a semigroup of nonlinear nonexpansive transformations on $S$.

The object of this paper is to obtain solutions to an evolution system in a general Banach space using the method of product integration. A definition of product integration is given as follows:

Suppose that $p$ is in $S, x>0$, and $z$ is a point in $S$ such that if $c>0$ there exists a chain $\left\{s_{i}\right\}_{i=0}^{m}$ from 0 to $x$ such that if $\left\{t_{i}\right\}_{i=0}^{n}$ is a refinement of $\left\{s_{i}\right\}_{i=0}^{m}$ then

$$
\left\|z-\prod_{i=1}^{n}\left(e-\left(t_{i}-t_{i-1}\right) A\right)^{-1} p\right\|<c .
$$

(Note that $e$ denotes the identity map on $S,\left(e-\left(t_{i}-t_{i-1}\right) A\right)^{-1}$ denotes the inverse map of $\left(e-\left(t_{i}-t_{i-1}\right) A\right), \prod_{i=1}^{1}\left(e-\left(t_{i}-t_{i-1}\right) A\right)^{-1} p=\left(e-\left(t_{1}-t_{0}\right) A\right)^{-1} p$, and if $j$ is an integer in $[2, n]$

$$
\prod_{i=1}^{j}\left(e-\left(t_{i}-t_{i-1}\right) A\right)^{-1} p=\left(e-\left(t_{j}-t_{j-1}\right) A\right)^{-1} \prod_{i=1}^{j-1}\left(e-\left(t_{i}-t_{i-1}\right) A\right)^{-1} p
$$

where the product operation is composition of mappings.) Then $z$ is said to be the product integral of $A$ with respect to $p$ from 0 to $x$ and is denoted by $\prod_{0}^{x}(e-d I A)^{-1} p$.

Received by the editors March 20, 1969 and, in revised form, August 20, 1969.

AMS Subject Classifications. Primary 3495, 3436; Secondary 3535, 3537.

Key Words and Phrases. Nonlinear evolution equations, product integration, dissipative mapping, semigroup of nonlinear nonexpansive transformations, infinitesimal generator.

Copyright (C) 1970, American Mathematical Society 
In [1] G. Birkhoff and in [16] J. Neuberger have used product integration to solve evolution systems where the mapping $A$ is Lipschitz continuous. In this paper the product integration method will be extended to mappings not necessarily Lipschitz continuous.

2. An existence theorem. Let $A$ be a mapping from a subset of $S$ to $S$ such that the following are true:

(I) $A$ is dissipative on its domain $D_{A}$, i.e., if $u, v \in D_{A}$ and $\varepsilon \geqq 0$ then $\|(e-\varepsilon A) u-(e-\varepsilon A) v\| \geqq\|u-v\|$.

(II) There is an open subset $C$ of $S$ such that $C \subseteq D_{A}$ and a positive number $\alpha$ such that if $0 \leqq \varepsilon<\alpha$ then $C \subseteq R_{(e-\varepsilon A)}$ (where $R_{(e-\varepsilon A)}$ denotes the range of $(e-\varepsilon A)$ ). (III) $A$ is continuous on $C$.

Note that by (I) if $\varepsilon>0$ then $(e-\varepsilon A)$ is $1-1$ on $D_{A}$ and by (II) if $0 \leqq \varepsilon<\alpha$ and $q \in C$ then $q \in D_{(e-\varepsilon A)^{-1}}=R_{(e-\varepsilon A)}$. If $0 \leqq \varepsilon<\alpha$ and $q \in R_{(e-\varepsilon A)}$ let $L(\varepsilon) q=(e-\varepsilon A)^{-1} q$. By (I) $L(\varepsilon)$ is nonexpansive on $R_{(e-\varepsilon A)}$, i.e., if $u, v \in R_{(e-\varepsilon A)}$ then

$$
\|L(\varepsilon) u-L(\varepsilon) v\| \leqq\|u-v\| .
$$

THEOREM. Let $A$ satisfy conditions (I), (II), and (III). If $p \in C$ and

$$
\gamma_{p}=\min \{\operatorname{dist}(p, \partial C) /\|A p\|, \alpha\},
$$

then there is a continuously differentiable function $g_{p}$ from $\left[0, \gamma_{p}\right)$ to $S$ such that $g_{p}(0)=p$ and if $0 \leqq x<\gamma_{p}, g_{p}^{\prime}(x)=A g_{p}(x)$ and $g_{p}(x)=\prod_{0}^{x}(e-d I A)^{-1} p$.

The theorem will be proved by means of a sequence of lemmas each of which is under the hypothesis of the theorem.

Lemma 1.1. If $q \in C$ and $0 \leqq x, y<\alpha$, then $\|L(x) q-L(y) q\| \leqq|x-y| \cdot\|A q\|$.

Proof. Using (2) we have that

$$
\begin{aligned}
\|L(x) q-L(y) q\| & =\|L(x) q-L(x)(e-x A) L(y) q\| \\
& \leqq\|q-(e-x A) L(y) q\| \\
& =\|q-[(x / y)(e-y A) L(y) q+(1-x / y) L(y) q]\| \\
& =|1-x / y|\|q-L(y) q\| \\
& \leqq|1-x / y|\|(e-y A) q-q\| \\
& =|x-y|\|A q\| .
\end{aligned}
$$

Lemma 1.2. Let $q \in C$, let $0<x<\gamma_{q}$, and let $\left\{s_{i}\right\}_{i=0}^{m}$ be a chain from 0 to $x$. If $j$ is an integer in $[1, m]$ then

$$
\begin{gathered}
\prod_{i=1}^{j-1} L\left(s_{i}-s_{i-1}\right) q \in C, \\
\left\|\prod_{i=1}^{j} L\left(s_{i}-s_{i-1}\right) q-q\right\| \leqq s_{j}\|A q\|,
\end{gathered}
$$


and

$$
\left\|A \prod_{i=1}^{j} L\left(s_{i}-s_{i-1}\right) q\right\| \leqq\|A q\|
$$

(Note that $\prod_{i=1}^{0} L\left(s_{i}-s_{i-1}\right)$ denotes the identity map, i.e., $\prod_{i=1}^{0} L\left(s_{i}-s_{i-1}\right) q=q$.)

Proof. The proof is by induction. For $j=1 \prod_{i=1}^{j-1} L\left(s_{i}-s_{i-1}\right) q=q \in C$,

$$
\left\|\prod_{i=1}^{1} L\left(s_{i}-s_{i-1}\right) q-q\right\| \leqq s_{1} \cdot\|A q\|
$$

(by Lemma 1.1), and

$$
\left\|A \prod_{i=1}^{1} L\left(s_{i}-s_{i-1}\right) q\right\|=\left\|1 / s_{1}\left[L\left(s_{1}-s_{0}\right) q-q\right]\right\| \leqq\|A q\| .
$$

Suppose that $j$ is an integer in $[1, m-1], \prod_{i=1}^{j-1} L\left(s_{i}-s_{i-1}\right) q \in C$,

$$
\left\|\prod_{i=1}^{j} L\left(s_{i}-s_{i-1}\right) q-q\right\| \leqq s_{j} \cdot\|A q\|
$$

and $\left\|A \prod_{i=1}^{j} L\left(s_{i}-s_{i-1}\right) q\right\| \leqq\|A q\|$. Then,

$$
\prod_{i=1}^{j} L\left(s_{i}-s_{i-1}\right) q \in C \subseteq D_{L_{\left(s_{j+1}-s_{j}\right)}}
$$

Further,

$$
\left\|\prod_{i=1}^{j+1} L\left(s_{i}-s_{i-1}\right) q-q\right\|=\left\|\sum_{i=1}^{j+1}\left[\prod_{k=i}^{j+1} L\left(s_{k}-s_{k-1}\right) q-\prod_{k=i+1}^{j+1} L\left(s_{k}-s_{k-1}\right) q\right]\right\|
$$

(note that $\prod_{k=j+2}^{j+1} L\left(s_{k}-s_{k-1}\right)$ is the identity map)

Moreover,

$$
\begin{aligned}
& \leqq \sum_{i=1}^{j+1}\left\|L\left(s_{i}-s_{i-1}\right) q-q\right\| \\
& \leqq s_{j+1} \cdot\|A q\| .
\end{aligned}
$$

$$
\begin{aligned}
\left\|A \prod_{i=1}^{j+1} L\left(s_{i}-s_{i-1}\right) q\right\| & =\left\|\left(\frac{1}{s_{j+1}-s_{j}}\right)\left[\prod_{i=1}^{j+1} L\left(s_{i}-s_{i-1}\right) q-\prod_{i=1}^{j} L\left(s_{i}-s_{i-1}\right) q\right]\right\| \\
& \leqq\left\|A \prod_{i=1}^{j} L\left(s_{i}-s_{i-1}\right) q\right\| \\
& \leqq\|A q\| .
\end{aligned}
$$

LEMMA 1.3. Let $q \in C$, let $0<x<\gamma_{q}$, and let $\left\{t_{i}\right\}_{i=0}^{n}$ be a chain from 0 to $x$. If $j$ is an integer in $[1, n]$ then

$$
\prod_{i=j}^{n} L\left(t_{i}-t_{i-1}\right) q-q=\sum_{i=j}^{n}\left(t_{i}-t_{i-1}\right) A \prod_{k=j}^{i} L\left(t_{k}-t_{k-1}\right) q .
$$


Proof.

$$
\begin{aligned}
\prod_{i=j}^{n} L\left(t_{i}-t_{i-1}\right) q-q & =\sum_{i=j}^{n}\left[\prod_{k=j}^{i} L\left(t_{k}-t_{k-1}\right) q-\prod_{k=j}^{i-1} L\left(t_{k}-t_{k-1}\right) q\right] \\
& =\sum_{i=j}^{n}\left(t_{i}-t_{i-1}\right) A L\left(t_{i}-t_{i-1}\right) \prod_{k=j}^{i-1} L\left(t_{k}-t_{k-1}\right) q \\
& =\sum_{i=j}^{n}\left(t_{i}-t_{i-1}\right) A \prod_{k=j}^{i} L\left(t_{k}-t_{k-1}\right) q .
\end{aligned}
$$

Let $p \in C$, let $c>0$, and let $m$ be a nonnegative integer. The number-sequence $\left\{s_{i}\right\}_{i=0}^{m}$ is said to have property $P_{c}$ provided that the following are true: (i) $s_{0}=0$, $s_{m}<\gamma_{p}$ (ii) $\left\{s_{i}\right\}_{i=0}^{m}$ is increasing, and (iii) if $h$ is an integer in [0,m-1], $s_{h} \leqq x \leqq s_{h+1}$, $\left\{t_{i}\right\}_{i=0}^{n}$ is a chain from $s_{h}$ to $x$, and $j$ is an integer in $[0, n]$, then

$$
\begin{aligned}
\| A \prod_{k=1}^{j} L\left(t_{k}-t_{k-1}\right) & \prod_{i=1}^{n} L\left(s_{i}-s_{i-1}\right) p \\
& -A \prod_{k=1}^{n} L\left(t_{k}-t_{k-1}\right) \prod_{i=1}^{n} L\left(s_{i}-s_{i-1}\right) p \| \leqq c .
\end{aligned}
$$

Lemma 1.4. Let $p \in C$, let $c>0$, and let $\left\{s_{i}\right\}_{i=0}^{m}$ have property $P_{c}$. There is a number $s_{m+1}$ such that $s_{m}<s_{m+1}<\gamma_{p}$ and $\left\{s_{i}\right\}_{i=0}^{m+1}$ has property $P_{c}$.

Proof. Lemma 1.4 follows from Lemma 1.2 and the continuity of $A$ at $\prod_{i=1}^{m} L\left(s_{i}-s_{i-1}\right) p$.

Lemma 1.5. Let $p \in C$, let $c>0$, and let $\left\{s_{i}\right\}_{i=0}^{m}$ have property $P_{c}$. Suppose that $y$ is a number such that $s_{m}<y<\gamma_{p}$ and if $s_{m+1}$ is a number such that $s_{m}<s_{m+1}<y$ then $\left\{s_{i}\right\}_{i=0}^{m+1}$ has property $P_{c}$. Then, if $s_{m+1}=y,\left\{s_{i}\right\}_{i=0}^{m+1}$ has property $P_{c}$.

Proof. Let $q=\prod_{i=1}^{m} L\left(s_{i}-s_{i-1}\right) p$, let $\left\{t_{i}\right\}_{i=0}^{n}$ be a chain from $s_{m}$ to $y$, and let $d>0$. There is a positive number $b$ such that if $u \in C$ and $\left\|u-\prod_{i=1}^{n} L\left(t_{i}-t_{i-1}\right) q\right\|<b$ then

$$
\left\|A u-A \prod_{i=1}^{n} L\left(t_{i}-t_{i-1}\right) q\right\|<d .
$$

There is a positive number $r$ such that $t_{n-1}<r<t_{n}=y$ and $t_{n}-r<b /\|A p\|$. By Lemmas 1.1 and 1.2

$$
\left\|L\left(r-t_{n-1}\right) \prod_{i=1}^{n-1} L\left(t_{i}-t_{i-1}\right) q-\prod_{i=1}^{n} L\left(t_{i}-t_{i-1}\right) q\right\| \leqq\left(t_{n}-r\right) \cdot\|A p\|<b .
$$

Then, if $j$ is an integer in $[0, n-1]$

$$
\begin{array}{rl}
\| A \prod_{i=1}^{j} L\left(t_{i}-t_{i-1}\right) q-A \prod_{i=1}^{n} & L\left(t_{i}-t_{i-1}\right) q \| \\
\leqq & \left\|A \prod_{i=1}^{j} L\left(t_{i}-t_{i-1}\right) q-A L\left(r-t_{n-1}\right) \prod_{i=1}^{n-1} L\left(t_{i}-t_{i-1}\right) q\right\| \\
& \quad+\left\|A L\left(r-t_{n-1}\right) \prod_{i=1}^{n-1} L\left(t_{i}-t_{i-1}\right) q-A \prod_{i=1}^{n} L\left(t_{i}-t_{i-1}\right) q\right\| \\
< & c+d .
\end{array}
$$


Then, if $j$ is an integer in $[0, n]$

$$
\left\|A \prod_{i=1}^{j} L\left(t_{i}-t_{i-1}\right) q-A \prod_{i=1}^{n} L\left(t_{i}-t_{i-1}\right) q\right\| \leqq c
$$

and so the lemma is established.

LemMA 1.6. Let $p \in C$, let $c>0$, and suppose that $\left\{s_{i}\right\}_{i=0}^{\infty}$ is an infinite increasing number-sequence such that $\lim \left\{s_{i}\right\}_{i=0}^{\infty}<\gamma_{p}$ and if $n$ is a nonnegative integer $\left\{s_{i}\right\}_{i=0}^{n}$ has property $P_{c}$. Then there is a positive integer $m$ and a sequence $\left\{r_{i}\right\}_{i=0}^{m+1}$ such that if $i$ is an integer in $[0, m] s_{i}=r_{i}, r_{m+1}=\lim \left\{s_{i}\right\}_{i=0}^{\infty}$, and $\left\{r_{i}\right\}_{i=0}^{m+1}$ has property $P_{c}$.

Proof. Let $q_{0}=p$ and if $n$ is a positive integer let $q_{n}=L\left(s_{n}-s_{n-1}\right) q_{n-1}$. If $n$ is a positive integer then $\left\|q_{n}-q_{n-1}\right\|=\left\|L\left(s_{n}-s_{n-1}\right) q_{n-1}-q_{n-1}\right\| \leqq\left(s_{n}-s_{n-1}\right) \cdot\|A p\|$. Let $s=\lim \left\{s_{i}\right\}_{i=0}^{\infty}$, let $q=\lim \left\{q_{i}\right\}_{i=0}^{\infty}$, and note that $q \in C$ since $\left\|q_{n}-p\right\|<s \cdot\|A p\|$ and so $\|q-p\|<\operatorname{dist}(p, \partial C)$. There is a positive number $b$ such that if $u \in C$ and $\|u-q\|<b$ then $\|A u-A q\|<c / 2$. Let $m$ be a positive integer such that $\left\|q-q_{m}\right\|<b / 2$ and $s-s_{m}<b / 2\|A p\|$. Let $0<x \leqq s-s_{m}$, let $\left\{t_{i}\right\}_{i=0}^{n}$ be a chain from 0 to $x$, and let $j$ be an integer in $[0, n]$. By Lemma 1.2

$$
\left\|\prod_{i=1}^{j} L\left(t_{i}-t_{i-1}\right) q_{m}-q_{m}\right\| \leqq t_{j} \cdot\|A p\|<b / 2
$$

and so

$$
\left\|A \prod_{i=1}^{j} L\left(t_{i}-t_{i-1}\right) q_{m}-A q\right\|<c / 2 .
$$

Then, if $j$ is an integer in $[0, n]$

$$
\begin{aligned}
\| A \prod_{i=1}^{j} L\left(t_{i}-t_{i-1}\right) q_{m}-A & \prod_{i=1}^{n} L\left(t_{i}-t_{i-1}\right) q_{m} \| \\
& \leqq\left\|A \prod_{i=1}^{j} L\left(t_{i}-t_{i-1}\right) q_{m}-A q\right\|+\left\|A q-A \prod_{i=1}^{n} L\left(t_{i}-t_{i-1}\right) q_{m}\right\| \\
& \leqq c
\end{aligned}
$$

and so the lemma is established.

Lemma 1.7. Let $p \in C$, let $c>0$, and let $0<x<\gamma_{p}$. There is a chain $\left\{s_{i}\right\}_{i=0}^{m}$ from 0 to $x$ such that $\left\{s_{i}\right\}_{i=0}^{m}$ has property $P_{c}$.

Proof. By Lemma 1.4 there is an infinite increasing number-sequence $\left\{s_{i}\right\}_{i=0}^{\infty}$ such that $\lim \left\{s_{i}\right\}_{i=0}^{\infty}<\gamma_{p}$ and if $n$ is a nonnegative integer $\left\{s_{i}\right\}_{i=0}^{n}$ has property $P_{c}$. Let $M$ denote the set of all such sequences. If $s=\left\{s_{i}\right\}_{i=0}^{\infty}$ is in $M$ let $z(s)$ denote the limit of $s$. If each of $s$ and $t$ belongs to $M$ define $s \leqq t$ only in case $s$ is $t$ or if $n$ is the greatest nonnegative integer such that if $i$ is an integer in $[0, n] s_{i}=t_{i}$, then $z(s) \leqq t_{n+1}$. Then, $\leqq$ is a partial ordering of $M$.

Assume that there exists no member $s$ of $M$ such that $z(s)>x$. Let $L$ be a linearly ordered subset of $M$ and let $y$ be the smallest positive number such that if $s$ is in 
$L, z(s) \leqq y$. Let $\left\{s_{i}(0)\right\}_{i=0}^{\infty},\left\{s_{i}(1)\right\}_{i=0}^{\infty}, \ldots$ be an increasing sequence of points in $L$ such that $z(s(0)), z(s(1)), \ldots$ converges to $y$. For each nonnegative integer $i$ define $y_{i}=\sup _{k} s_{i}(k)$. Then, $y_{i} \leqq y_{i+1}$ and $\lim \left\{y_{i}\right\}_{i=0}^{\infty}=y$.

Suppose there is a positive integer $n$ such that $y_{n}=y$. Then there is a least positive integer $n$ such that $y_{n}=y$ and there must exist an integer $k$ such that $s_{i}(k)=s_{i}(j)$ for each integer $i$ in $[0, n-1]$ and $j \geqq k$. In this case $s_{n}(k), s_{n}(k+1), \ldots$ converges to $y$ and so by Lemma $1.5\left\{s_{i}\right\}_{i=0}^{n}, s_{i}=s_{i}(k)$ for $i$ in $[0, n-1]$ and $s_{n}=y$, has property $P_{c}$. Further, since $y<\gamma_{p}$, we have by Lemma 1.4 that $\left\{s_{i}\right\}_{i=0}^{n}$ may be extended to a member $\left\{s_{i}\right\}_{i=0}^{\infty}$ of $M$ and so $\left\{s_{i}\right\}_{i=0}^{\infty}$ is an upper bound for $L$. If there is no positive integer $n$ such that $y_{n}=y$ then $y_{n}<y$ for every $n,\left\{y_{n}\right\}_{n=0}^{\infty}$ is in $M,\left\{y_{n}\right\}_{n=0}^{\infty} \geqq s(k)$ for every $k$, and thus $\left\{y_{n}\right\}_{n=0}^{\infty}$ is an upper bound for $L$.

Thus, if $L$ is a linearly ordered subset of $M$, then $L$ is bounded by a member of $M$. By Zorn's lemma there exists $u \in M$ such that $u$ is maximal. But then we have a contradiction since $z(u) \leqq x<\gamma_{p}$ and by Lemma 1.6 there exists $t \in M$ such that $u<t$. Hence, there exists $s \in M$ such that $z(s)>x$ and the lemma is proved.

LemMa 1.8. Let $p \in C$, let $c>0$, and let $0<x<\gamma_{p}$. There is a chain $\left\{s_{i}\right\}_{i=0}^{m}$ from 0 to $x$ such that if $\left\{t_{i}\right\}_{i=0}^{n}$ is a refinement of $\left\{s_{i}\right\}_{i=0}^{m}$ then

$$
\left\|\prod_{i=1}^{n} L\left(t_{i}-t_{i-1}\right) p-\prod_{i=1}^{m} L\left(s_{i}-s_{i-1}\right) p\right\|<c .
$$

Proof. Let $\left\{s_{i}\right\}_{i=0}^{m}$ be a chain from 0 to $x$ such that $\left\{s_{i}\right\}_{i=0}^{m}$ has property $P_{c}$. Let $\left\{t_{i}\right\}_{i=0}^{n}$ be a refinement of $\left\{s_{i}\right\}_{i=0}^{m}$, i.e., there is an increasing sequence $u$ such that $u_{0}=0, u_{m}=n$, and if $i$ is an integer in $[0, m] s_{i}=t_{u_{i}}$. If $i$ is an integer in $[1, m]$ let $K_{i}=\prod_{j=u_{i-1}+1}^{u_{i}} L\left(t_{j}-t_{j-1}\right)$, let $J_{i}=\prod_{j=1}^{i} L\left(s_{j}-s_{j-1}\right)$, let $K_{m+1}=e$, and let $J_{0}=e$. Then,

$$
\begin{array}{rl}
\| \prod_{i=1}^{n} & L\left(t_{i}-t_{i-1}\right) p-\prod_{i=1}^{m} L\left(s_{i}-s_{i-1}\right) p \| \\
& =\left\|\prod_{i=1}^{m} K_{i} p-J_{m} p\right\| \\
& =\left\|\sum_{i=1}^{m}\left[\prod_{j=i}^{m} K_{j} J_{i-1} p-\prod_{j=i+1}^{m} K_{j} J_{i} p\right]\right\| \\
& \leqq \sum_{i=1}^{m}\left\|K_{i} J_{i-1} p-J_{i} p\right\| \\
& =\sum_{i=1}^{m}\left\|K_{i} J_{i-1} p-L\left(s_{i}-s_{i-1}\right) J_{i-1} p\right\| \\
& \leqq \sum_{i=1}^{m}\left\|\left(e-\left(s_{i}-s_{i-1}\right) A\right) K_{i} J_{i-1} p-J_{i-1} p\right\| \\
& =\sum_{i=1}^{m}\left\|\left[\prod_{j=u_{i}-1+1}^{u_{i}} L\left(t_{j}-t_{j-1}\right) J_{i-1} p-J_{i-1} p\right]-\left(s_{i}-s_{i-1}\right) A K_{i} J_{i-1} p\right\|
\end{array}
$$




$$
\begin{aligned}
& =\sum_{i=1}^{m}\left\|\sum_{j=u_{i-1}+1}^{u_{i}}\left(t_{j}-t_{j-1}\right)\left[A \prod_{k=u_{i-1}+1}^{j} L\left(t_{k}-t_{k-1}\right) J_{i-1} p-A K_{i} J_{i-1} p\right]\right\| \\
& \leqq \sum_{i=1}^{m} \sum_{j=u_{i-1}+1}^{u_{i}}\left(t_{j}-t_{j-1}\right) \| A \prod_{k=u_{i-1}+1}^{j} L\left(t_{k}-t_{k-1}\right) J_{i-1} p \\
& \leqq c \cdot \sum_{i=1}^{m} \sum_{j=u_{i-1}+1}^{u_{i}}\left(t_{j}-t_{j-1}\right) \\
& =c \cdot x .
\end{aligned}
$$

Proof of the theorem. Let $p \in C$. If $x=0$, then $\prod_{0}^{x}(e-d I A)^{-1} p=p$. If $0<x<\gamma_{p}$, then $\prod_{0}^{x}(e-d I A)^{-1} p$ exists by virtue of Lemma 1.8. If $0 \leqq x<\gamma_{p}$ define $g_{p}(x)$ $=\prod_{0}^{x}(e-d I A)^{-1} p$. By Lemma 1.2 we see that $g_{p}$ is Lipschitz continuous on $\left[0, \gamma_{p}\right)$ with Lipschitz constant $\leqq\|A p\|, g_{p}(x) \in C$ for $x \in\left[0, \gamma_{p}\right)$, and $\left\|A g_{p}(x)\right\| \leqq\|A p\|$ for $x \in\left[0, \gamma_{p}\right)$. For $0 \leqq x<\gamma_{p}$ we have that dist $(p, \partial C) \leqq \operatorname{dist}\left(g_{p}(x), \partial C\right)+\left\|p-g_{p}(x)\right\|$ $\leqq$ dist $\left(g_{p}(x), \partial C\right)+x\|A p\|$. Hence,

$$
\begin{aligned}
\operatorname{dist}(p, \partial C) /\|A p\| & \leqq \operatorname{dist}\left(g_{p}(x), \partial C\right) /\|A p\|+x \\
& \leqq \operatorname{dist}\left(g_{p}(x), \partial C\right) /\left\|A g_{p}(x)\right\|+x
\end{aligned}
$$

and so $\gamma_{p}-x \leqq \gamma_{g_{p}(x)}$. Thus, if $0 \leqq x<\gamma_{p}$ and $0 \leqq y<\gamma_{p}-x$, one sees that $g_{g_{p}(x)}(y)$ $=g_{p}(x+y)$. To show that $g_{p}^{\prime}=A g_{p}$ let $0 \leqq x<\gamma_{p}$ and let $c>0$. By Lemma 1.2 there is a positive number $z<\gamma_{p}-x$ such that if $0<y<z$ and $\left\{s_{i}\right\}_{i=0}^{m}$ is a chain from 0 to $y$, then

$$
\left\|A \prod_{i=1}^{m} L\left(s_{i}-s_{i-1}\right) g_{p}(x)-A g_{p}(x)\right\|<c / 2 .
$$

Let $0<y<z$. There is a chain $\left\{t_{i}\right\}_{i=0}^{n}$ from 0 to $y$ such that

$$
\left\|\prod_{i=1}^{n} L\left(t_{i}-t_{i-1}\right) g_{p}(x)-g_{g_{p}(x)}(y)\right\|<c \cdot y / 2 .
$$

Then,

$$
\begin{aligned}
\| \frac{1}{y}\left[g_{p}(x+y)-g_{p}(x)\right] & -A g_{p}(x) \| \\
& <\frac{c}{2}+\frac{1}{y}\left\|\left(\prod_{i=1}^{n} L\left(t_{i}-t_{i-1}\right) g_{p}(x)-g_{p}(x)\right)-y A g_{p}(x)\right\| \\
& =\frac{c}{2}+\frac{1}{y}\left\|\sum_{i=1}^{n}\left(t_{i}-t_{i-1}\right) A \prod_{j=1}^{i} L\left(t_{j}-t_{j-1}\right) g_{p}(x)-y A g_{p}(x)\right\| \\
& \leqq \frac{c}{2}+\frac{1}{y} \sum_{i=1}^{n}\left(t_{i}-t_{i-1}\right)\left\|A \prod_{j=1}^{i} L\left(t_{j}-t_{j-1}\right) g_{p}(x)-A g_{p}(x)\right\| \\
& <c
\end{aligned}
$$


and so $g_{p}^{\prime+}(x)=A g_{p}(x)$. Thus, $g_{p}^{\prime+}=A g_{p}$ on $\left[0, \gamma_{p}\right)$ and so $g_{p}$ has a continuous right derivative on $\left[0, \gamma_{p}\right)$. Then $g_{p}$ has a continuous derivative on $\left[0, \gamma_{p}\right)$ and so the theorem is proved.

Corollary. Let $A$ be a mapping from the Banach space $S$ to $S$ such that the following are true:

$\left(\mathrm{I}^{\prime}\right) A$ is dissipative on $S$, i.e., if $u, v \in D_{A}$ and $\varepsilon \geqq 0$ then $\|(e-\varepsilon A) u-(e-\varepsilon A) v\|$ $\geqq\|u-v\|$

(II') $R_{(e-\varepsilon A)}=S$ for each $\varepsilon \geqq 0$

(III') $A$ is continuous on $S$.

If $p \in S$ then there is a continuously differentiable function $g_{p}$ from $[0, \infty)$ to $S$ such that $g_{p}(0)=p$ and if $x \geqq 0 g_{p}^{\prime}(x)=A g_{p}(x)$ and $g_{p}(x)=\prod_{0}^{x}(e-d I A)^{-1} p$.

Proof. The proof follows immediately from the theorem if one observes that $\alpha=+\infty$ and dist $(p, \partial S)=+\infty$.

It may be noted that a result of J. Dorroh [8] can be used to show that the solutions of $g_{p}^{\prime}=A g_{p}, g_{p}(0)=p$ in the corollary are unique. In [15] G. Minty has shown that if $S$ is the Hilbert space then ( $\left.\mathrm{I}^{\prime}\right)$ and (III') imply (II'). More generally, it has been shown recently by $\mathrm{T}$. Kato in [11] that (I') and (III') imply (II') in the case that $S^{*}$ is uniformly convex. If $S$ is a general Banach space $\mathrm{F}$. Browder has shown in [4] that ( $\left.\mathrm{I}^{\prime}\right)$ and (III') imply ( $\left.\mathrm{II}^{\prime}\right)$ in the case that $A$ is locally uniformly continuous.

By virtue of the corollary one may define for each $x \geqq 0$ the transformation $T(x)$ from $S$ to $S$ as follows: $T(x) p=g_{p}(x)$ for each $p \in S$. Then $T$ is a strongly continuous semigroup of nonlinear nonexpansive transformations on $S$, i.e.,

(i) $T(x+y)=T(x) T(y)$ for $x, y \geqq 0$,

(ii) $T(0)=e$,

(iii) $\|T(x) p-T(x) q\| \leqq\|p-q\|$ for $x \geqq 0$ and $p, q \in S$ and

(iv) $g_{p}(x)=T(x) p$ is continuous for $p$ fixed and $x \geqq 0$.

Further, $A$ is the infinitesimal generator of $T$, i.e., $A p=g_{p}^{\prime+}(0)$ for each $p \in S$. In [2], [14], [17], [18], and [19] representations are given for nonlinear nonexpansive semigroups of transformations in terms of their infinitesimal generators using product integrals.

3. Examples. In conclusion we give some examples. In [6] a well-known example is given by $\mathrm{J}$. Dieudonné of a continuous mapping $A$ from a Banach space $S$ to $S$ for which there is no solution to the equation $g^{\prime}=A g$ and $g(0)=\overline{0}$. This example is given in a Banach space which is not reflexive. Recently, J. Yorke [20] has given an example of a continuous mapping $A$ from a Hilbert space to itself for which no solution exists to $g^{\prime}=A g, g(0)=\overline{0}$.

In the examples below the mapping $A$ satisfies conditions ( $\left.\mathrm{I}^{\prime}\right),\left(\mathrm{II}^{\prime}\right)$, and (III') of the corollary.

ExAmple 1. Let $S=E_{1}$ and let $A$ be a continuous nonincreasing function from $E_{1}$ to $E_{1}$. 
ExAmple 2. Let $S=C_{[0,1]}$, i.e., $S$ is the Banach space of continuous real-valued functions on $[0,1]$ with supremum norm. Let $F$ be a continuous increasing function from $E_{1}$ onto $E_{1}$ such that $F^{\prime}$ is continuous and nonincreasing on $E_{1}$. Define the mapping $A$ on $C_{[0,1]}$ as follows:

$$
A f=F^{\prime}\left[F^{-1}[f]\right] \text { for each } f \in C_{[0,1]} .
$$

The solutions $g_{f}$ of the corollary are then given by $g_{f}(x)=F\left[x+F^{-1}[f]\right]$ for $x \geqq 0$.

In both Examples 1 and $2 A$ may be neither linear nor Lipschitz continuous. In both, however, $A$ is locally uniformly continuous. In Example 3 the mapping $A$ is not locally uniformly continuous.

ExAmple 3. Let $S=\left(c_{0}\right)$, i.e., $S$ is the Banach space of real-number sequences $x=\left(x_{n}\right)$ converging to 0 with $\|x\|=\sup _{n}\left|x_{n}\right|$. If each of $(a, b)$ and $(c, d)$ is a point in the plane define the function $F_{[(a, b),(c, d)]}$ from $[a, c]$ to $[b, d]$ by

$$
F_{[(a, b),(c, d)]}(x)=b+\left(\frac{d-b}{c-a}\right)(x-a) \text { for } x \in[a, c] .
$$

For each positive integer $n$ define the function $A_{n}$ from $E_{1}$ to $E_{1}$ as follows:

$$
\begin{aligned}
A_{n}(x)= & \text { if } x<-1 \\
& =0 \quad \text { if } x \geqq 0 \\
& =F_{[(-1 / k, 1 / k),(-1 / k+(1 / n)[1 / k-1 /(k+1)], 1 /(k+1)]}(x) \quad \text { if } x \in\left[-\frac{1}{k},-\frac{1}{k}+\frac{1}{n}\left(\frac{1}{k}-\frac{1}{k+1}\right)\right) \\
& =\frac{1}{k+1} \text { if } x \in\left[-\frac{1}{k}+\frac{1}{n}\left(\frac{1}{k}-\frac{1}{k+1}\right),-\frac{1}{k+1}\right) \quad k=1,2, \ldots
\end{aligned}
$$

Define the mapping $A$ from $\left(c_{0}\right)$ to $\left(c_{0}\right)$ by $A x=\left(A_{n}\left(x_{n}\right)\right)$ for each $x=\left(x_{n}\right) \in\left(c_{0}\right)$. One sees that $A$ satisfies conditions ( ('), (II'), and (III'), since for each positive integer $n A_{n}$ is nonincreasing and continuous. Moreover, there is no neighborhood about $\overline{0}$ on which $A$ is uniformly continuous.

\section{REFERENCES}

1. Garrett Birkoff, On product integration, J. Mathematical Phys. 16 (1937), 104-132.

2. H. Brezis and A. Pazy, Semigroups of nonlinear contractions on covex sets (to appear).

3. F. E. Browder, Nonlinear equations of evolution, Ann. of Math. (2) 80 (1964), 485-523. MR 30 \#4167.

4. - Nonlinear operators and nonlinear equations of evolution in Banach spaces, Proc. Sympos. Pure Math., vol. 18, Amer. Math. Soc., Providence, R. I. (to appear).

5. M. G. Crandall and A. Pazy, Nonlinear semi-groups of contractions and dissipative sets, J. Functional Analysis 3 (1969), 376-418.

6. J. Dieudonné, Foundations of modern analysis, Pure and Appl. Math., vol. 10, Academic Press, New York, 1960. MR 22 \#11074.

7. J. R. Dorroh, Integral equations in normed abelian groups, Pacific J. Math. 13 (1963), 1143-1158. MR 28 \#1469. 
8. J. R. Dorroh, Some classes of semi-groups of nonlinear transformations and their generators, J. Math. Soc. Japan 20 (1968), 437-455. MR 37 \#6796.

9. E. Hille and R. S. Phillips, Functional analysis and semi-groups, rev. ed., Amer. Math. Soc. Colloq. Publ., vol. 31, Amer. Math. Soc., Providence, R. I., 1957. MR 19, 664.

10. T. Kato, Nonlinear semigroups and evolution equations, J. Math. Soc. Japan 19 (1967), 508-520. MR 37 \#1820.

11. - Accretive operators and nonlinear evolution equations in Banach spaces, Proc. Sympos. Pure Math., vol. 18, Amer. Math., Soc., Providence, R. I. (to appear).

12. Y. Kömura, Nonlinear semi-groups in Hilbert space, J. Math. Soc. Japan 19 (1967), 493-507. MR 35 \#7176.

13. - Differentiability of nonlinear semi-groups, J. Math. Soc. Japan 21 (1969), 375-402.

14. J. Mermin, Accretive operators and nonlinear semi-groups, Thesis, Univ. of California, Berkeley, 1968.

15. G. J. Minty, Monotone (nonlinear) operators in Hilbert space, Duke Math. J. 29 (1962), 341-346. MR 29 \#6319.

16. J. W. Neuberger, Continuous products and nonlinear integral equations, Pacific J. Math. 8 (1958), 529-549. MR 21 \#1509.

17. - An exponential formula for one-parameter semi-groups of nonlinear transformations, J. Math. Soc. Japan 18 (1966), 154-157. MR 34 \#622.

18. S. Oharu and I. Miyadera, Approximation of semi-groups of nonlinear operators (to appear).

19. G. F. Webb, Representation of nonlinear nonexpansive semi-groups of transformations in Banach space, J. Math. Mech. 19 (1969), 159-170.

20. J. Yorke, A continuous differential equation in Hilbert space without existence (to appear).

\section{VANDERBILT UNIVERSITY, NASHVILLE, TeNNesSEe}

